\title{
Tratamento cirúrgico de ameloblastoma sólido convencional: relato de caso clínico
}

\author{
Surgical treatment of conventional solid ameloblastoma: \\ clinical case report
}

\author{
Radamés Bezerra Melo* \\ Nayara Cristina Monteiro Carneiro* \\ Wallyson Luis Maues da Fonseca** \\ Jader Fadul de Lima*** \\ Helen Patrícia da Silva Araújo ${ }^{* * * *}$ \\ Hélder Antônio Rebelo Pontes ${ }^{* * * * *}$
}

\section{Resumo}

O ameloblastoma convencional é uma neoplasia benigna originada do tecido epitelial odontogênico encontrado nos ossos maxilares. É caracterizado, clinicamente, como tumor de crescimento lento, na maioria dos casos, é assintomático ou pode causar parestesias, assim como pode ocasionar deslocamento, mobilidade e reabsorção dental. Histologicamente, a variante multicística apresenta diversos padrões, como: folicular, plexiforme e desmoplásico. Devido a essas variações histopatológicas, existem diversos métodos de tratamento, desde a enucleação e curetagem até ressecções mais agressivas, sendo que essas são bem mais indicadas, tendo em vista o potencial agressivo e infiltrativo da lesão. Objetivo: este trabalho visa relatar um caso clínico de ameloblastoma multicístico tratado por ressecção marginal mandibular. Relato de caso: paciente do gênero feminino, 40 anos de idade, com hipótese diagnóstica de ameloblastoma por meio de radiografia panorâmica de rotina. Por intermédio da biópsia incisional, foi diagnosticado ameloblastoma multicístico em região de corpo, ângulo e ramo mandibular esquerdo. O tratamento de escolha foi, assim como na maioria dos casos relatados na literatura, a ressecção cirúrgica. Considerações finais: essa opção de tratamento é justificada pelo alto índice de recidiva das lesões tratadas de forma mais conservadora. No presente trabalho, a paciente foi mantida em proservação por trinta meses, período em que não foi observado nenhum sinal de recidiva da lesão ou complicações pós-operatórias.

Palavras-chave: Ameloblastoma. Neoplasias. Terapêutica. Tumores odontogênicos.

\section{Introdução}

O ameloblastoma é um tumor odontogênico benigno, originado de restos de tecidos epiteliais da embriogênese $^{1}$. Por ser de crescimento lento, invasivo e, geralmente, assintomático, na maioria dos casos, é descoberto após exames radiográficos de rotina ${ }^{2}$. A literatura considera três variantes dessa neoplasia, com aspectos clínicos e radiográficos divergentes, classificadas em: ameloblastoma sólido convencional ou multicístico, unicístico e periférico ou extraósseo ${ }^{3}$, com prevalência distribuída na ordem de $86 \%, 13 \%$ e $1 \%$, em todos os casos de ameloblastoma ${ }^{1}$.

O ameloblastoma sólido convencional é encontrado em grande variação etária, com prevalência dos 30 aos 70 anos e sem predileção por gênero ${ }^{1,3}$. É

Mestre e doutorando em Cirurgia pela Universidade Federal do Ceará. Residência em Cirurgia e Traumatologia Bucomaxilofacial pelo Hospital Universitário João de Barros Barreto, Universidade Federal do Pará, Belém, Pará, Brasil. Professor do curso de Odontologia da Faculdade Paulo Picanço, Fortaleza, Ceará, Brasil.

Residente em Cirurgia e Traumatologia Bucomaxilofacial do Hospital Universitário João de Barros Barreto, Universidade Federal do Pará, Belém, Pará, Brasil. Acadêmico de Odontologia da Universidade Federal do Pará. Estagiário do Serviço de Cirurgia e Traumatologia Bucomaxilofacial do Hospital Universitário João de Barros Barreto, Universidade Federal do Pará, Belém, Pará, Brasil.

**** Cirurgião e Traumatologista Bucomaxilofacial pelo Hospital Universitário Pedro Ernesto. Preceptor da Residência de Cirurgia e Traumatologia Bucomaxilofacial do Hospital Universitário João de Barros Barreto, Universidade Federal do Pará, Belém, Pará, Brasil.

Cirurgiã-dentista pela Universidade Federal do Pará, Belém, Pará, Brasil.

***** Doutor em Patologia Bucal pela Universidade de São Paulo. Coordenador da Residência em Cirurgia e Traumatologia Bucomaxilofacial do Hospital Universitário João de Barros Barreto, Universidade Federal do Pará, Belém, Pará, Brasil. 
classificado, clinicamente, como o tipo de ameloblastoma mais agressivo, por apresentar alta capacidade infiltrativa entre as trabéculas ósseas ${ }^{4,5}$. Trata-se de tumor assintomático e de evolução lenta, que chega a atingir grandes proporções sem apresentar dor e parestesia ${ }^{1,6}$.

A descrição radiográfica da lesão, na maioria dos casos, é uma radiolucidez multilocular com aspecto de "bolhas de sabão" ou "favos de mel", de margens irregulares ${ }^{3}$. Presença de reabsorção radicular em dentes adjacentes e expansão de cortical óssea são comumente observadas. Em alguns casos, nota-se a lesão envolvendo um terceiro molar inferior inclu$\mathrm{so}^{1,7}$.

A lesão, nos aspectos histopatológicos, subdivide-se em: folicular, plexiforme, acantomatoso, de células granulares, de células basais e desmoplásico, sendo os dois primeiros mais encontrados ${ }^{3,4}$. Ademais, o índice de recidiva elevado dessa neoplasia está diretamente ligado às suas variantes histológicas, que se infiltram através das trabéculas ósseas dessa lesão ${ }^{8}$.

A depender do tamanho da lesão, há várias formas de tratamento para o ameloblastoma sólido, que variam de uma abordagem conservadora até outras mais invasivas, como a enucleação seguida de curetagem, na presença ou não do uso da técnica de crioterapia, ressecção marginal ou em bloco e hemimandibulectomia .

Dessa forma, este artigo tem por objetivo apresentar um caso clínico de ameloblastoma multicístico, bem como a história da doença, suas características radiográficas, histopatológicas e seu tratamento.

\section{Relato de caso}

Paciente do gênero feminino, com 40 anos de idade, compareceu ao Serviço de Cirurgia e Diagnósticos de Patologia Oral do Hospital Universitário João de Barros Barreto (Belém, Pará), com hipótese diagnóstica de ameloblastoma por meio de radiografia panorâmica de rotina. Foi realizada biópsia incisional, na qual foi diagnosticado ameloblastoma multicístico em região de corpo, ângulo e ramo mandibular esquerdo. Ao ser realizado exame extraoral, observou-se simetria facial, ausência de tumefação e limitação de abertura bucal, sem relatos de dor ou edema (Figura 1).

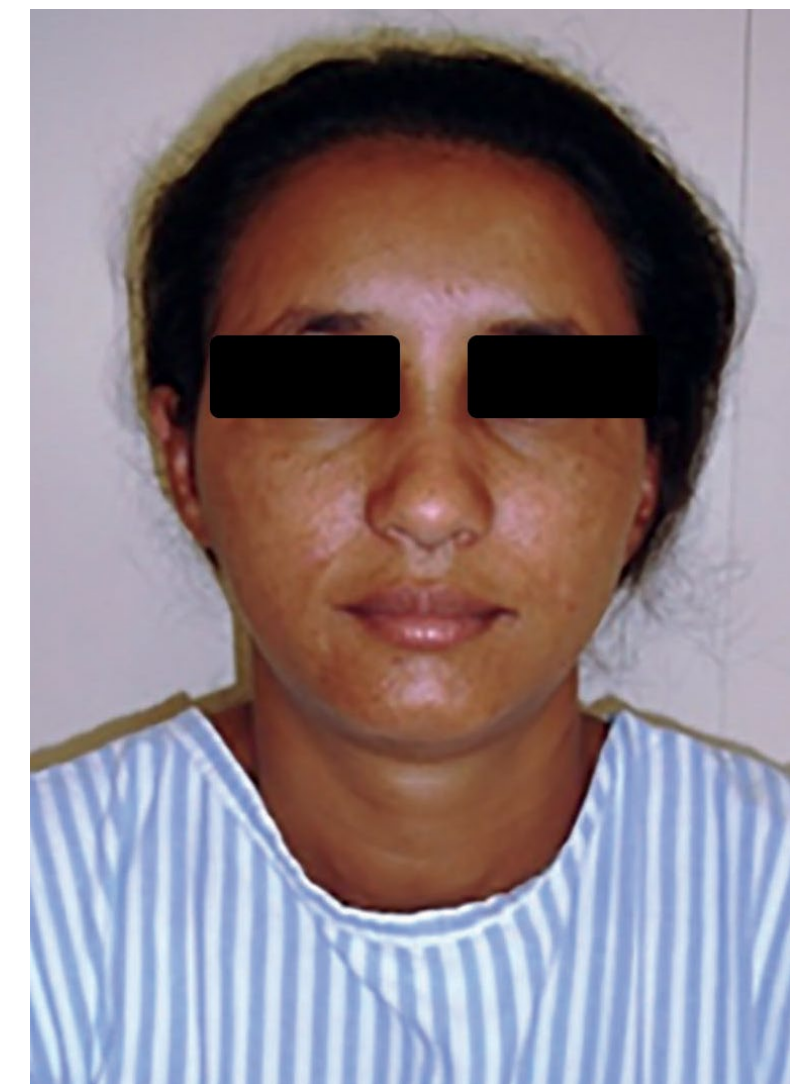

Figura 1 - Aspecto extraoral pré-operatório

Fonte: dos autores.

Ao serem realizados os exames radiográfico e tomográfico, ficou evidenciada presença de lesão radiolúcida, com aspecto multilocular em região de corpo, ângulo e ramo mandibular esquerdo (Figura $2 \mathrm{~A} \mathrm{e} \mathrm{B)}$.

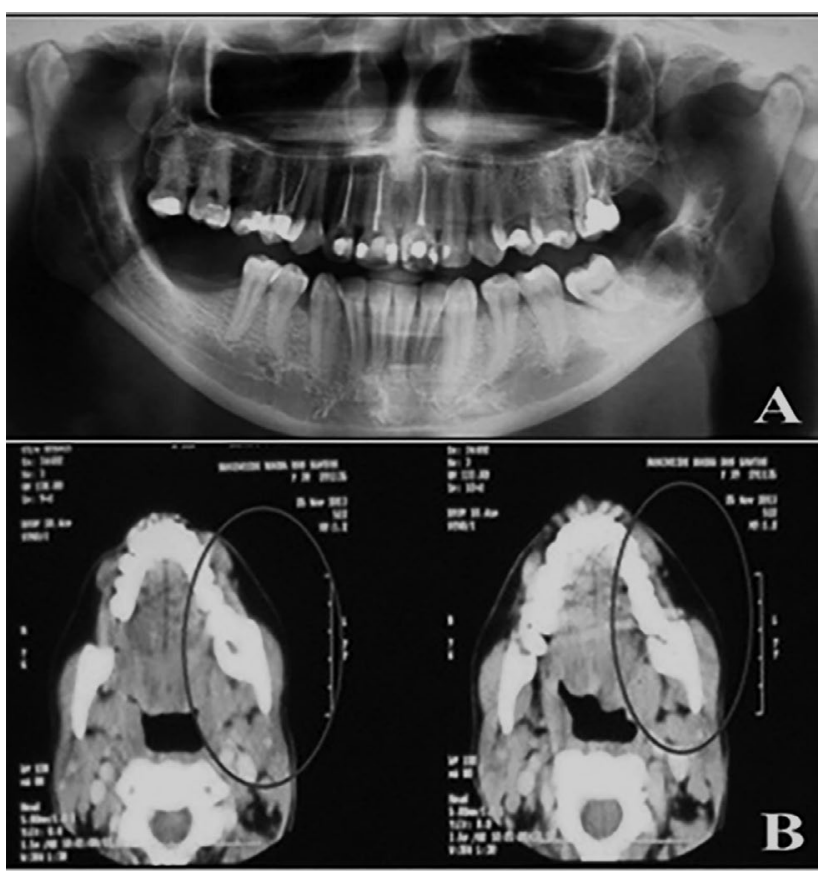

Figura 2 - A) Exame radiográfico revelando presença de lesão radiolúcida multilocular no lado esquerdo do paciente e $B$ exame tomográfico evidenciando envolvimento de corpo, ângulo e ramo mandibular esquerdo, com perda de continuidade óssea, indicado nos círculos

Fonte: dos autores. 
Paciente foi submetida à internação para cirurgia programada no Hospital Divina Providência, situado no município de Marituba, região metropolitana de Belém do Pará. Optou-se pela ressecção cirúrgica mandibular marginal como tratamento, preconizando uma margem de segurança de $1 \mathrm{~cm}$, realizado pela equipe do programa de residência em Cirurgia e Traumatologia Bucomaxilofacial do Hospital Universitário João de Barros Barreto (Figura 3).

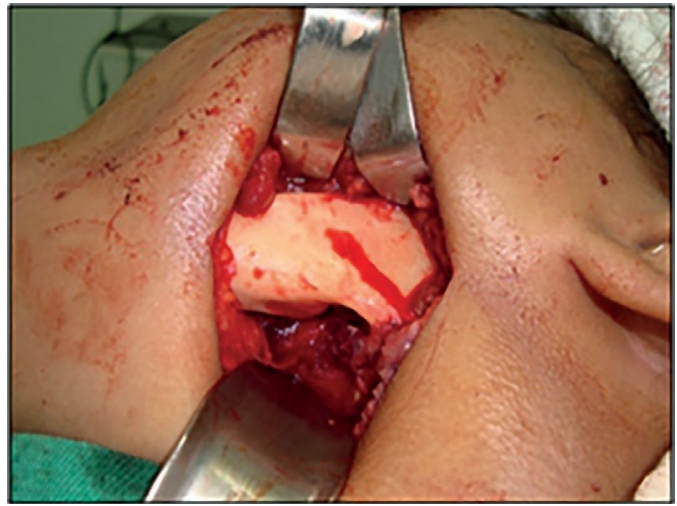

Figura 3 - Acesso submandibular ou de Risdon para exploração de lesão em corpo, ângulo e ramo mandibular

Fonte: dos autores.

Após exérese total da lesão, realizou-se a preservação da basilar óssea da mandíbula, como preconizado na literatura, que foi sustentada e fixada com placa de titânio de reconstrução do sistema de $2,4 \mathrm{~mm}$, com o objetivo de manter os contornos estéticos normais e os aspectos funcionais do sistema estomatognático da paciente (Figura 4).

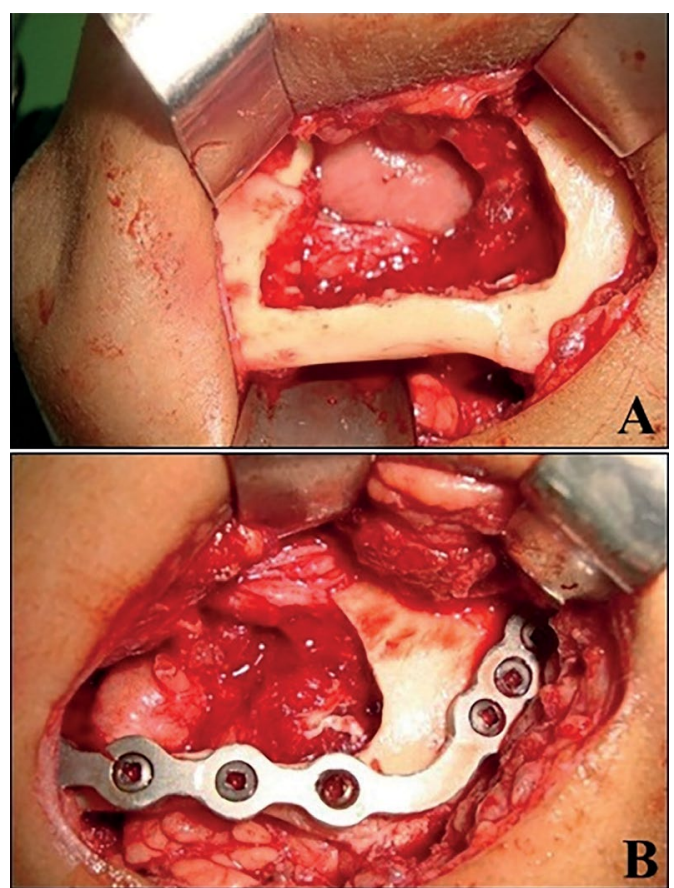

Figura 4 - A) Remoção total da lesão com margem de seguranca e manutenção da basilar óssea para maior estabilização e fixação da placa de titânio e B) fixação com placa de titânio de reconstrução do sistema 2,4 mm

Fonte: dos autores.
A peça cirúrgica foi submetida a novo exame histopatológico, confirmando assim o diagnóstico primário de ameloblastoma multicístico ou sólido convencional (Figura 5).

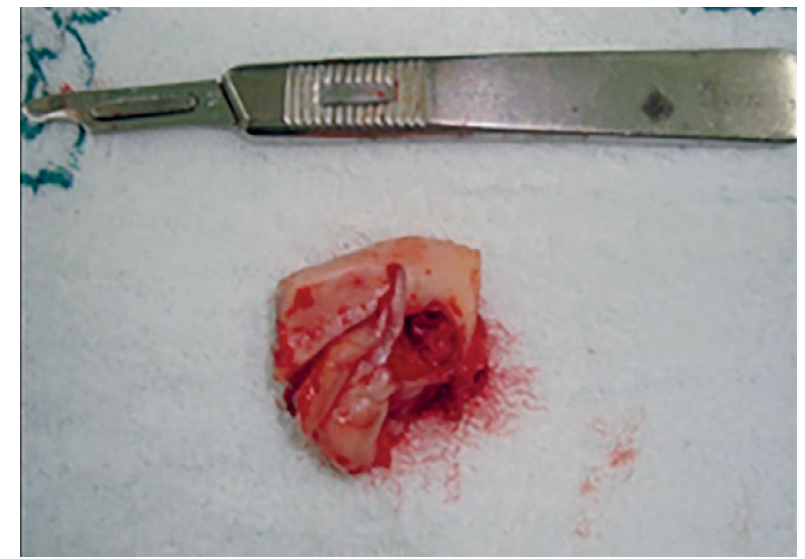

Figura 5 - Imagem da peça cirúrgica enviada ao exame histopatológico

Fonte: dos autores.

Nos exames histopatológicos da lesão, os aspectos encontrados estão de acordo com os relatados na literatura para o ameloblastoma sólido convencional. Foi observado o padrão folicular, com ilhotas de epitélio, provavelmente, provenientes do órgão do esmalte em meio a um estroma de tecido conjuntivo fibroso. Também, foram observadas células colunares semelhantes a ameloblastos cercando a região central dessas ilhas, em que se visualiza a formação de cistos dentro dessas ilhas de epitélio. Os núcleos dessas células encontram-se no lado oposto à membrana basal, caracterizando polaridade reversa, característica típica desse padrão histológico de ameloblastoma multicístico. Não existe diferença de tratamento para os subtipos de ameloblastoma multicístico, de acordo com a literatura (Figura 6).

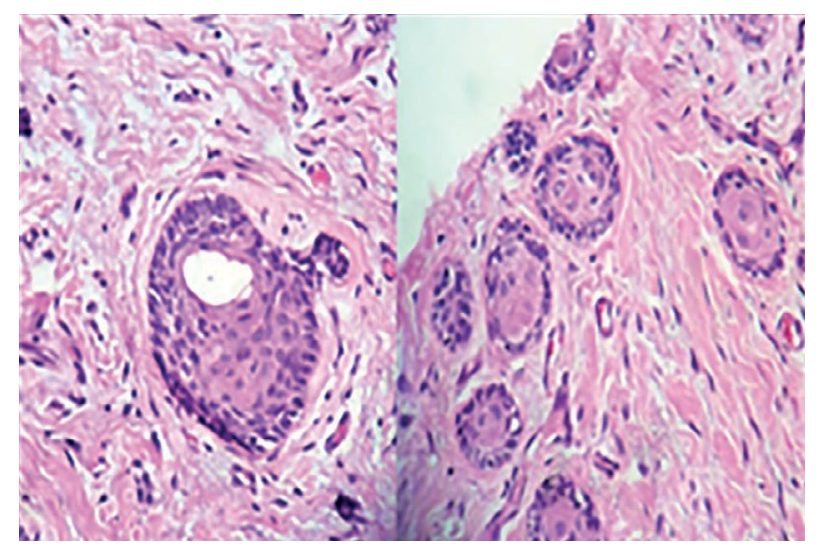

Figura 6 - Imagem histopatológica da lesão confirmando o diagnóstico inicial de ameloblastoma de padrão multicístico

Fonte: dos autores.

Após trinta meses da intervenção cirúrgica, não foi observado nenhum sinal possível de recidiva da lesão, como pode ser observado nos exames de ima- 
gem em reconstrução 3D (Figura 7) e nas imagens extraorais (Figura 8). A paciente evoluiu sem sinais de recidivas ou complicações pós-operatórias.

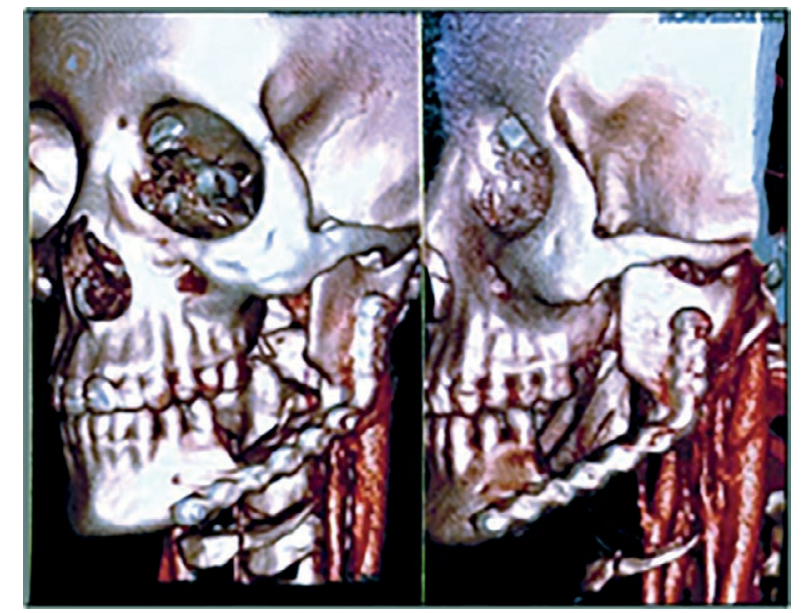

Figura 7 - Reconstrução 3D em plano 3/4 e perfil

Fonte: dos autores.
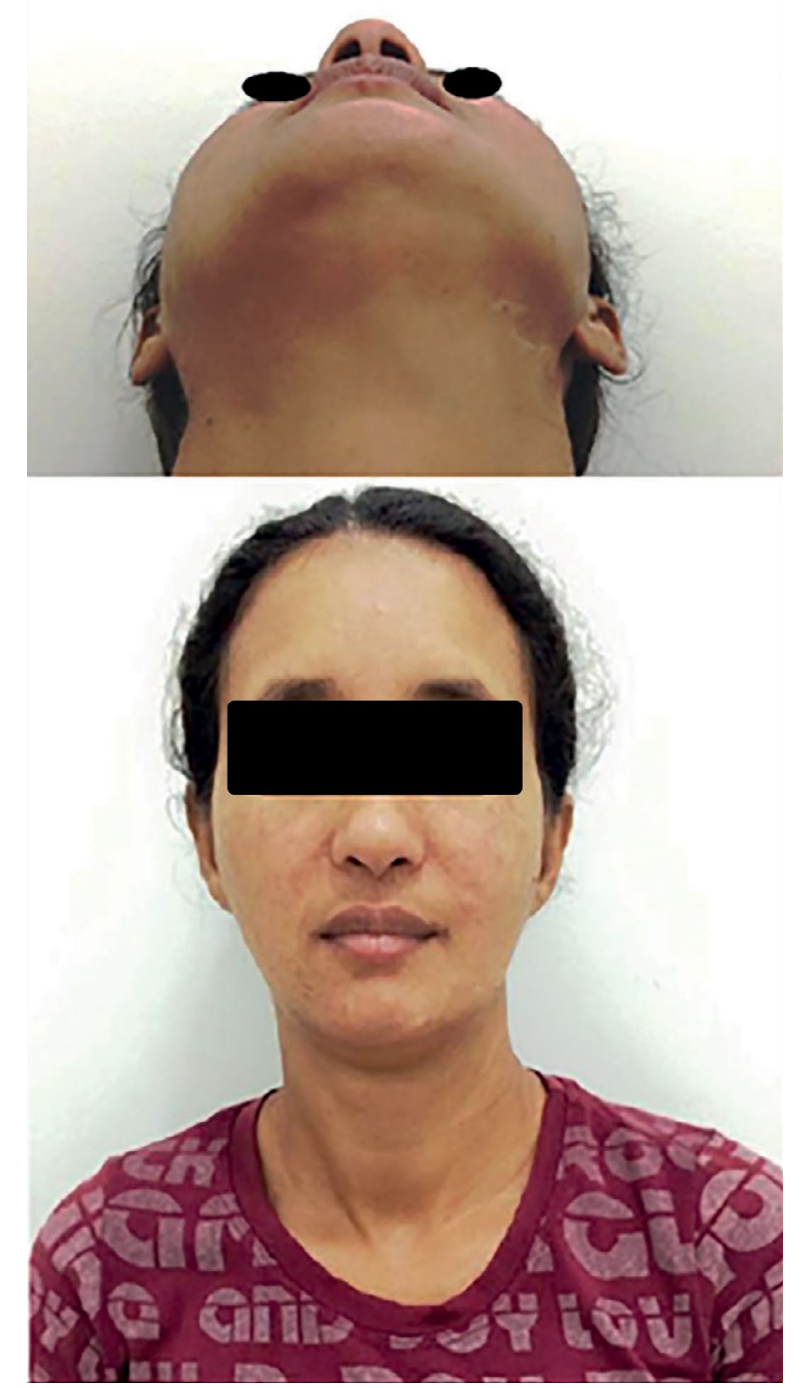

Figura 8 - Imagens extraorais apresentando cicatrização satisfatória da incisão pós-cirúrgica e ausência de tumefações e/ou assimetria facial

Fonte: dos autores.

\section{Discussão}

O ameloblastoma é considerado a neoplasia mais frequente, correspondendo a $1 \%$ dentre os cistos e tumores odontogênicos, e pode ocorrer em qualquer região de maxila e mandíbula ${ }^{1,2}$. No entanto, estudos apontam que 99,1\% dos casos ocorrem na mandíbula, em região do corpo, ramo e ângulo, o que corrobora com o caso em questão ${ }^{2,3}$. A predileção por algum gênero é controversa, havendo discordâncias em muitos estudos ${ }^{6,7}$

Radiograficamente, é comum o ameloblastoma multicístico apresentar-se como uma lesão de aspecto semelhante a "bolhas de sabão", para lesões com loculações maiores, e com aspecto de "favo de mel"10, quando menores.

Nos aspectos histopatológicos, o padrão celular do ameloblastoma sólido convencional consiste, em resumo, na proliferação de células epiteliais, cujas características císticas variam em sua apresentação, dependendo do subtipo histológico ${ }^{11}$.

Apesar de várias formas de tratamento já terem sido largamente debatidas na literatura, ainda não há um consenso sobre qual seria a mais indicada. Um dos motivos para essa controvérsia é o fato de essa neoplasia ser uma lesão com alto poder infiltrativo dentro das trabéculas ósseas, o que dificulta a visualização adequada de seus limites em exames de imagem. Por esse motivo, é necessário o uso de exames mais específicos, como a tomografia computadorizada. Esses exames de imagens permitem a visualização mais precisa das margens do tumor e, consequentemente, a elaboração de um plano de tratamento mais eficaz para cada caso. No caso relatado, a realização da tomografia foi de grande importância para o fechamento do plano de tratamento e também durante a realização da abordagem cirúrgica, na qual foi removido o tumor com uma margem de segurança precisa ${ }^{11-14}$.

Quando a enucleação da lesão seguida de curetagem é preconizada, expõe-se o paciente a um alto índice de recidiva, que pode chegar a $90 \%$ na mandíbula e a $100 \%$ na maxila ${ }^{6,11}$. A ressecção marginal ou em bloco é mais utilizada por conter baixas taxas de recidivas e promover remoção total do tumor, pois se emprega nessa técnica uma margem de segurança de $1 \mathrm{~cm}$ a $1,5 \mathrm{~cm}$ além dos limites da lesão ${ }^{1,15}$, procedimento que foi empregado no caso relatado. Até o momento, observa-se o sucesso do tratamento, uma vez que não há qualquer sinal de recidiva ao longo de dois anos e meio de acompanhamento.

Contudo, deve-se levar em consideração o melhor tipo de tratamento e o mais eficaz para determinado padrão de lesão, o que requer do cirurgião o conhecimento dos problemas associados às intervenções radicais, que podem ser: disfunção mastigatória, mutilações, deformidades faciais e movimentação anormal da mandíbula ${ }^{1,16,17}$. 


\section{Considerações finais}

O tratamento de escolha para o ameloblastoma, na maioria dos casos, é a ressecção cirúrgica. Essa opção de tratamento é a mais preconizada devido ao alto índice de recidiva das lesões tratadas de forma mais conservadora. $\mathrm{O}$ presente trabalho relatou um caso de ressecção marginal ou segmentar mandibular com acompanhamento de trinta meses sem quaisquer sinais de recidiva.

\section{Abstract}

The conventional ameloblastoma is a benign tumor originating from the odontogenic epithelial tissue found in maxillary bones. It is characterized clinically as a slow-growing tumor, and in most cases, it is asymptomatic or may cause paresthesia, as well as dental displacement, mobility, and resorption. Histologically, the multicystic variant has different patterns, such as follicular, plexiform, and desmoplastic. Due to these histopathological changes, there are several treatment methods, ranging from enucleation and curettage to more aggressive resections. These are much more recommended because of the aggressive and infiltrative potential of lesions. Objective: This paper aims to describe a clinical case of multicystic ameloblastoma treated by marginal mandibular resection. Case report: Female patient, 40 years old, diagnosed with ameloblastoma by routine panoramic radiograph. The incisional biopsy provided a diagnosis of multicystic ameloblastoma in the body region, angle to the left mandibular ramus. The treatment of choice was, as in most cases reported in the literature, surgical resection. Final considerations: This treatment option is more justified by the high rate of recurrence of lesions treated more conservatively. In the present study, the patient was kept under proservation for 30 months and no signs of recurrence of the lesion or postoperative complications were observed.

Keywords: Ameloblastoma. Odontogenic tumors. Therapeutics. Maxillomandibular tumors.

\section{Referências}

1. Neville BW, Dam DD, Allen CM, Bouquot JE. Patologia Oral \& Maxilofacial. Rio de Janeiro: Guanabara Koogan; 2004.

2. Corrêa APS, Brust AWA, Jesus GP. Prototipagem rápida: um método auxiliar no tratamento de ameloblastoma - relato de caso. Rev Odontol Unesp 2010; 39(4):247-54.

3. Gomes ACA, Silva EDO, Albert DGM, Lira MFC, Andrade ESS. Conceito atual no tratamento dos ameloblastomas. Rev Bras Cir Traumatol Buco-maxilo-fac 2006; 6:9-16.

4. Grempel RG, Gaião L, Souza WD, Sobreira T. Tendências de abordagens cirúrgicas no tratamento de ameloblastomas. Rev Bras Patol Oral 2003; 2:13-7.

5. Fregnani E, Faria P, Rangel A, Almeida O, Vargas P. Tumores odontogênicos: análise de 113 casos da Faculdade de Odontologia de Piracicaba-Unicamp. Rev Pós Grad 2003; 10(4):355-9.
6. Hatada K, Noma H, Katakura A, Yama M, Takano M, Ide Y, et al. Clinicostatistical study of ameloblastoma treatment. Bull Tokio Dent Coll 2001; 42(2):87-95.

7. Gomes ACA, e Silva EDO, Albert DGM, de Lira MFC, Andrade ESS. Conceito atual no tratamento dos ameloblastomas. Rev Bras Cir Traumatol Buco-maxilo-fac 2006; 6(3):9-16.

8. Gomes ACA, Dias E, Gomes DO, Paraíso DP, Nascimento GJF, Cabral RAA. Ameloblastoma: tratamento cirúrgico conservador ou radical? Rev Bras Cir Traumatol Buco-maxilo-Fac 2002; 2(2):17-24.

9. Raldi UFV, Guimarães-Filho R, Moraes MB, Neves ACC. Tratamento de ameloblastoma. Rev Gauch Odontol 2010; 58(1):123-6.

10. Chapelle KAOM, Stoelinga PJW, de Wilde PCM, Brouns JJA, Voorsmit RACA. Rational approach to diagnosis and treatment of ameloblastomas and odontogenic keratocysts. Br J Oral Maxillofac Surg 2004; 42(5):381-90.

11. Nakamura N, Higuchi Y, Mitsuyasy T, Sandra F, Ohishi N. Comparison of long-term results between different approaches to ameloblastoma. Oral Surg Oral Med Oral Pathol Oral Radiol Endod 2003; 93:13-20.

12. Medeiros M, Porto GG, Laureano Filho R, Portela L, Vaconcellos RH. Ameloblastoma em mandíbula. Rev Bras Otorrinolaringol 2008; 74:478.

13. Alvarenga RL, Chrcanovic BR, Horta MCR, Souza LN, Maia BF. Ameloblastoma multiquístico mandibular tratado com terapia menos invasiva: caso clínico y revisión de la literatura. Rev Esp Cirug Oral y Maxilofac 2010; 32(4):172-7.

14. Martins MD, Rosa AO, Martins MAT, Bussadori AS, Fernandes KPS. Ameloblastoma: revisão de literatura. ConScientiae Saúde 2007; 6(2):269-78.

15. Yazdi I, Seyedmajidi M, Foroughi R. Desmoplastic ameloblastoma (a hybrid variant): report of a case and review of the literature. Arch Iran Med 2009; 12(3):304-8.

16. Sham E, Leong J, Maher R, Schenberg M, Leung M, Mansour AK. Mandibular ameloblastoma: clinical experience and literature review. ANZ J Surg 2009; 79(10):739-44.

17. Mendonça JC, Santos AA, Lopes HB. Hemimaxilectomia associada à crioterapia no tratamento de ameloblastomas: relato de caso. Rev Soc Bras Cir Craniomaxilof 2011; 14(1):63-6.

Endereço para correspondência:

Wallyson Luis Maues da Fonseca

Hospital Universitário João de Barros Barreto

Rua dos Mundurucus, 4.487

66073-000 Belém, PA

Telefone: (91) 98921-2928/ (91) 98460-4076

E-mail: wallysonlmfonseca@gmail.com

Recebido: 14/03/2016. Aceito: 27/05/2016. 